\title{
Recognizing toxic species in aquatic habitats: a potential concern in lake management
}

\author{
Mikołaj KOKOCINSKI and Harold G. MARSHALL ${ }^{1)}$ \\ Department of Hydrobiology, Adam Mickiewicz University, Poznań, Poland \\ ${ }^{1)}$ Department of Biological Sciences, Old Dominion University, Norfolk, 23529 Virginia, U.S.A.
}

\begin{abstract}
The importance of distinguishing toxic and non-toxic algal species is becoming a more common problem for management decisions associated with various freshwater and estuarine habitats. An example is given where two dinoflagellates, originally unidentified as closely resembling the toxin producing Pfiesteria spp., have been compared to these species. In order to clarify any relationship to Pfiesteria spp., scanning electron microscopy was used to determine the plate tabulation patterns of these dinoflagellates and make comparisons to the tabulation present in Pfiesteria spp. The results indicated significant differences in the plate tabulations of these taxa to distinguish them from Pfiesteria spp.
\end{abstract}

Key words: Pfiesteria sp., toxicity, aquatic management, plate tabulation

\section{INTRODUCTION}

Potentially toxic species may cause management problems in lakes and other aquatic habitats. However, there may be occasions when it is difficult to determine the identification of these species when there is close morphological similarity between toxic and non-toxic taxa. Early identification in the presence of any toxin producing species would be beneficial to management awareness and response to their presence before toxic conditions develop. This would allow a timely and appropriate management decision to be made regarding access and/or usage of a particular lake and its natural resources, and any control measures that could be taken to reduce the impact of a toxic event.

An example is provided here regarding situations when morphologically similar species of toxin and nontoxin producing species may occur in the same habitat, and need to be identified to determine which species is present. Within the phytoplankton community there are numerous taxa possessing similar morphological characteristics and size. Among many of these dinoflagellates using light microscopy alone will not be satisfactory to distinguish these species from each other. This identification can only be confirmed using electron microscopy or/and genetic molecular protocols (e.g. polymerase chain reaction analysis if available). By using scanning electron microscopy in examining the armored plate-covered dinoflagellates, the tabulation patterns of the plates that encircle the vegetative cells can be identified. Each dinoflagellate taxon has a unique pattern, shape, and number of these plates. In this way, differences in the tabulation patterns associated with toxin producing species may be compared to other similar dinoflagellates to determine if they are the toxic species.
In this study comparisons of the external morphological characteristics (plate tabulation) of toxin producing $P f i$ esteria spp. are made to similar appearing dinoflagellates of questionable identity, using scanning electron microscopy.

There are two recognized Pfiesteria species ( $P$. piscicida, $P$. shumwayae) that have been identified, and these have produced bloom concentrations in estuaries along the United States east coast. They are both heterotrophic and mixotrophic with a complex life cycle that includes motile, cyst, and amoeboid forms. In the presence of fish they can produce a water soluble toxin that can result in extensive fish kills and cause human illness (Burkholder et al. 2001). Often found in the same waters as Pfiesteria spp. are similar appearing dinoflagellates. These have been generally referred to as Pfiesteria-like organisms (PLO) and possess life cycle stages, size, and morphological features similar to Pfiesteria piscicida and P. shumwayae (Marshall et al. 2000). Using only light microscopy, mistaken identifications are likely, leading to confusion and mis-information presented to those in management positions.

Many of the PLO have been established in cultures, but have not been fully identified, and their relationship to the Pfiesteria species not fully known. One area that is lacking in many of these unidentified PLO is the determination of their complete plate tabulation, for comparison to Pfiesteria spp., and other dinoflagellates. Lacking a specific nomenclature for these PLO when isolated for culture, investigators have assigned a numerical reference, or a term generally associated with its collections site to identify these cells. For instance, one PLO category is known as the "Lucy" group (or complex) (Steidinger et al. 2001), others by numbers assigned to them, e.g. CCMP1838. 


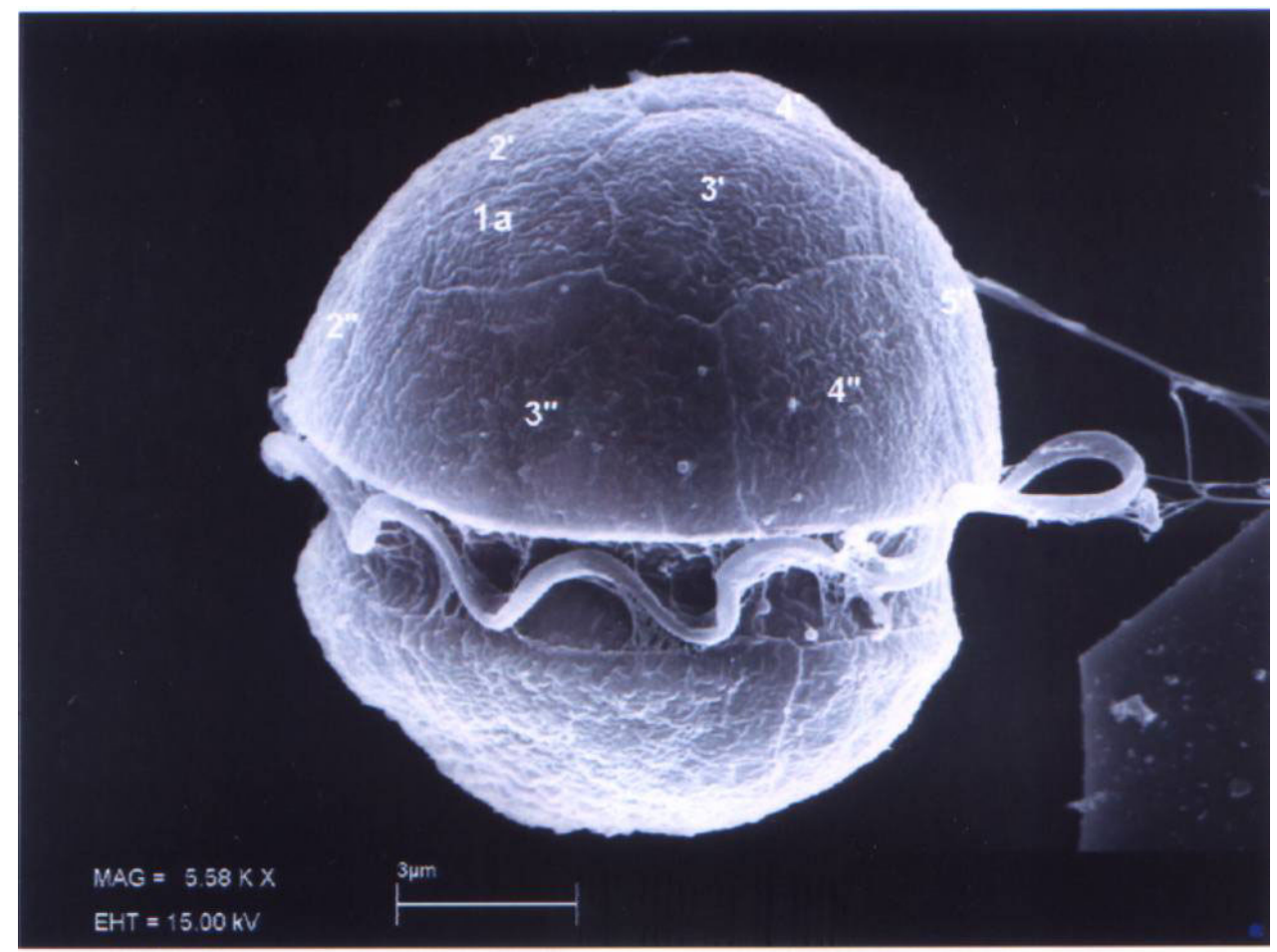

Fig. 1. Dorsal view of Pfiesteria shumwaye.

The objectives of this study are to: 1) Examine the plate tabulation of a Pfiesteria-like organism (cell culture CCMP 1838) with unknown plate tabulation to determine if it is a Pfiesteria species, and 2) Make comparisons of the plate tabulations and features of CCMP 1838 to: a.) Cells of another dinoflagellate culture CCMP 1833 (a member of this PLO "Lucy" group), and b) cells of Pfiesteria shumwayae (a toxic dinoflagellate).

\section{METHODS}

Cell cultures of dinoflagellate strains CCMP1833 and CCMP1838, identified as Pfiesteria-like organisms, were obtained from the Provasoli-Guillard National Center for Culture of Marine Phytoplankton (CCMP). A culture of Pfiesteria shumwayae was provided by Dr. JoAnn Burkholder (North Carolina State University). Laboratory sub-cultures of these taxa were established in our laboratory and fed Rhodomonas sp. (CCMP-757) in filtered and autoclaved seawater (10-15 ppt), in an incubator at $24.5^{\circ} \mathrm{C}$ and a 12:12 hour light:dark regime. A suture-swelling technique (Truby 1997) was used in the preparation of cells from each of these cultures for scanning electron microscope examination. The fixative included the combination of $1.5 \%$ glutaraldehyde, $1 \%$ osmium tetroxide, and sodium cocodylate. Cells were fixed from 15-40 minutes in the dark and at $4{ }^{\circ} \mathrm{C}$, followed by rinsing in $0.1 \mathrm{M}(6.8-7.3 \mathrm{pH})$ buffer, and dehydrogenated in a graded ethanol series. The fixed cells were critical-point dried, rinsed, and sputter coated with gold-palladium, and examined with a Leo model 435 VP scanning electron microscope.

\section{RESULTS}

Plate tabulation formulae were determined for the two PLO taxa CCMP1833 and CCMP1838, and comparisons were made to the plate characteristics of Pfiesteria shumwayae and Pfiesteria piscicida (Steidinger et al. 2001). Both taxa had a similar apical pore complex, and the plate patterns for CCMP1833 and CCMP1838 were similar, being: 4', 2a, 6', 6c, 2s, 5',', 0p, 2',',. However, there was a distinct difference in the size of the diamond-shaped intercalary (a) plates. In CCMP1838, these anterior intercalaries $(1 \mathrm{a}, 2 \mathrm{a})$ are much larger than in CCMP1833. Also, the two taxa differ in cell length, with CCMP1833 $<15 \mu \mathrm{m}$ and CCMP1838 $>15 \mu \mathrm{m}$. The plate tabulation of Pfiesteria shumwayae is 4', 1a, 6", 6c, 4s, 5",, 0p, 2",' (Burkholder et al. 2001) (Fig. 1). This pattern differs from the two CCMP taxa by having only one rectangular, diamond shaped anterior accessory plate (a) instead two rectangular, diamond shaped anterior accessory plates (1a, 2a) located at the left and right side of the 3' plate as observed in CCMP1833 and CCMP1838 (Fig. 2).

Plate formula of those two PLO taxa differs also from plate tabulation of Pfiesteria piscicida (Burkholder et al. 2001). This plate tabulation was 4', 1a, 5', 6c, 4s, 5',',0p, 2 ",', This pattern differs from two CCMP taxa by having one much smaller than $P$. shumwayae triangular, diamond shaped anterior intercalary plate and only 5 instead of 6 precingular plates. Subsequent genetic analysis using polymerase chain reaction (PCR) analysis indicated CCMP 1838 did not test positive for Pfiesteria, nor did a preliminary fish bioassay indicate it was toxic. 


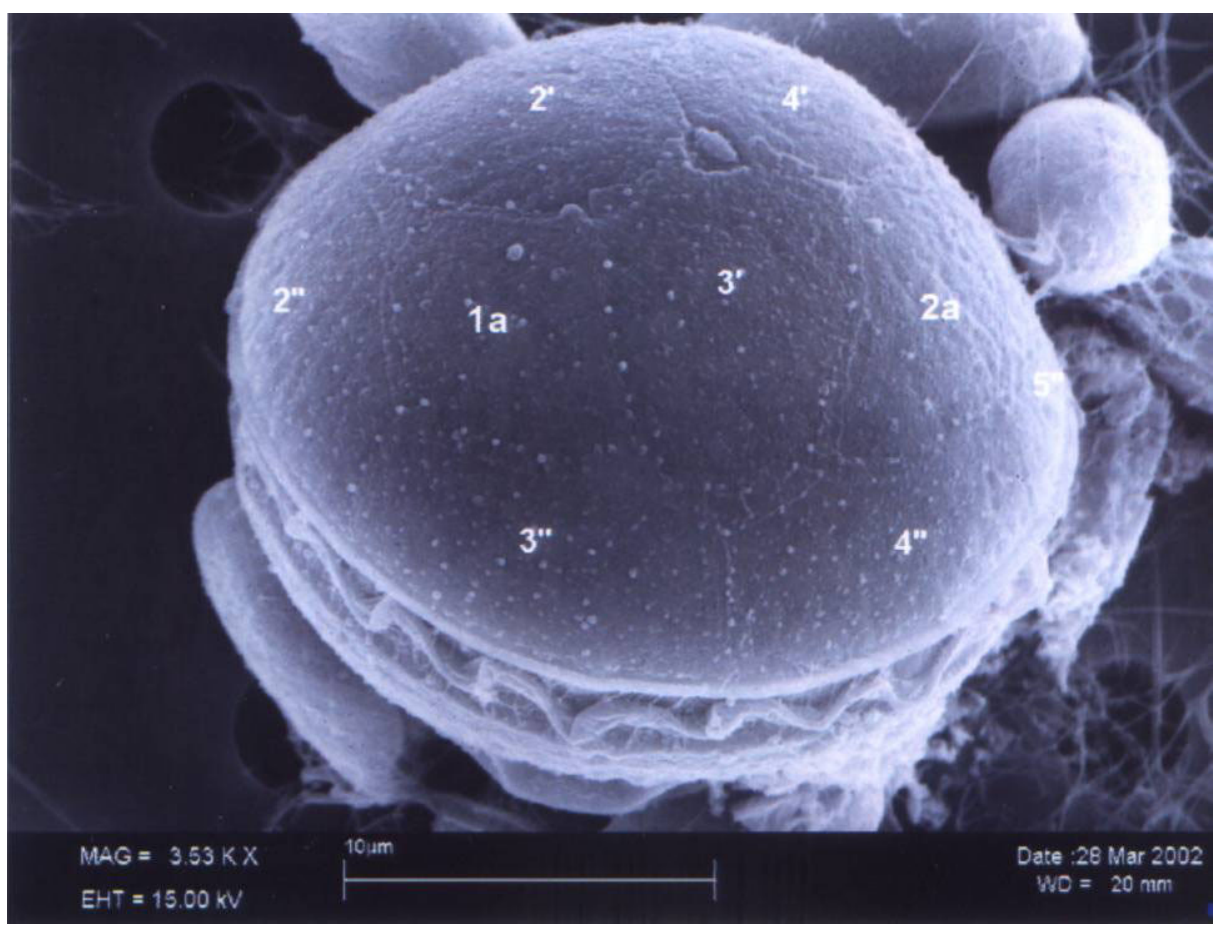

Fig. 2. Epithecal view of strain $\# 1838$. Note anterior intercalaries No. 1a, 2a

\section{CONCLUSIONS}

1. A protocol is presented to distinguish unknown dinoflagellates, with similar morphological features (e.g. size, shape) to Pfiesteria spp., from these toxic dinoflagellates.

2. Neither CCMP1833, nor CCMP1838 had plate patterns and tabulations similar to $P$. shumwayae or $P$. piscicida, and are not considered Pfiesteria spp.

3. CCMP1833 and CCMP1838 had similar plate tabulations. The only observed differences between the two taxa were the larger cell size and larger anterior intercalaries (1a, 2a) present in CCMP1838.

4. Although both CCMP1833 and CCMP1838 have the plate tabulation of the "Lucy" group, the size characteristics of CCMP1838 makes it a more representative taxon for this category. Both of these cultures represent taxa that require further classification.

5. Accurate identification of suspect toxin producing taxa is a valuable asset in making management decisions and in this situation identifies new taxa that should be further investigated for any potential ability to produce toxins.

\section{ACKNOWLEDGMENTS}

Appreciation is extended to the Virginia Department of Health and the U.S. Center for Disease Control and
Prevention for support of this study that is a component of a broader monitoring program of Pfiesteria spp. and Pfiesteria-like organisms in Virginia estuaries. Special thanks are given to Regan Taylor and Cheryl Walton for their assistance during various phases of this study, to Brian Dyer for providing information about species toxicity, and Denise Wadsworth for her assistance during SEM examination.

\section{REFERENCES}

Burkholder, J., H. Glasgow \& N. Deamer. 2001. Overview and present status of toxic Pfiesteria complex (Dinophyceae). Phycologia, 40(3): 186-214.

Marshall, H., A. Gordon, D. Seaborn, B. Dyer, W. Dunstan \& M. Seaborn. 2000. Comparative culture and toxicity studies between the toxic dinoflagellate Pfiesteria piscicida and a morphologically similar dinoflagellate. J. Experimental Marine Biology and Ecology, 255: 5174.

Steidinger, K., J. Landsberg, R. Richardson, E. Truby, B. Blakesley, P. Scott, P. Tester, T. Tengs, P. Mason, S. Morton, D. Seaborn, W. Litaker, K. Reece, D. Oldach, L. Haas, \& G. Vasta. 2001. Classification and identification of Pfiesteria and Pfiesteria-like Species. Environmental Health Perspectives, 109(5): 661-665.

Truby, E.W. 1997. Preparation of single-celled marine dinoflagellates for electron microscopy. Microscopy Research and Technique, 36: 337-340. 